\title{
EARLY CHILDHOOD ALLERGY LINKED WITH DEVELOPMENT OF ATTENTION DEFICIT HYPERACTIVITY DISORDER AND AUTISM SPECTRUM DISORDER
}

\author{
Shay Nemet ${ }^{1}$, Ilan Asher ${ }^{2}$, Israel Yoles ${ }^{3}$, Tuvia Baevsky ${ }^{3}$, and Zev Sthoeget ${ }^{1}$ \\ ${ }^{1}$ Kaplan Medical Center \\ ${ }^{2}$ Kupat Holim Clalit \\ ${ }^{3}$ Clalit Health Services
}

January 3, 2022

\begin{abstract}
Background: Previous studies reported controversial results regarding the association between allergic disorders and ADHD/ASD. The aim of this article is to investigate whether allergic disorders are associated with ADHD/ASD in a large cohort of pediatric patients. Methods: A retrospective study using the pediatric (0-18 year) database (ICD-9-CM codes) of Clalit Health Services during the years (2000-2018). Diagnosis of all disorders was made by specialist physicians. Results: 117,022 consecutive non-selective allergic children diagnosed with one or more allergic disorder (asthma, rhinitis. conjunctivitis, skin, food, or drug allergy) and 116,968 non-allergic children were enrolled to our study. The mean follow-up period was $11 \pm 6$ years. The presence of allergic disorders in early childhood (mean age of allergic diagnosis $4.5 \pm 4.3$ years) in boys as well as in girls, significantly increased the risk to develop ADHD (O.R 2.45, CI 2.39-2.51; P<0.0001), ASD (O.R 1.17, CI 1.08-1.27; P $<0.0001$ ) or both ADHD+ASD (O.R 1.5, CI 1.35-1.79; $\mathrm{P}<0.0001)$. Children with more than one allergic comorbidity revealed a much higher risk. In a multivariable analysis (adjusted for age at study entry, number of yearly visits and gender) the risk of allergic children to develop ADHD and ADHD+ASD, but not ASD alone, remained significantly higher. Conclusion: Allergic disorder in early childhood significantly increased the risk to develop ADHD, and to a less extend ASD, in later life.
\end{abstract}

\section{EARLY CHILDHOOD ALLERGY LINKED WITH DEVELOPMENT OF ATTENTION DE- FICIT HYPERACTIVITY DISORDER AND AUTISM SPECTRUM DISORDER}

\section{LIST OF AUTHORS:}

Shay Nemet, MD, Clinical Immunology Allergy and AIDS Center, Kaplan Medical Center, Rehovot, Israel. shaynemeth@gmail.com

Israel Yoles, MD, Clalit Health Services, Central District, Israel. yoles@netvision.net.il

Tuvia Baevsky, MD, Clalit Health Services, Central District, Israel. tbaevsky@gmail.com

Zev Sthoeger, MD, Clinical Immunology Allergy and AIDS Center, Kaplan Medical Center, Rehovot, Israel. sthoeger@gmail.com

CORRESPONDING AUTHOR:

Ilan Asher, MD, Clinical Immunology Allergy and AIDS Center, Kaplan Medical Center, 1 Pasternak Rd, Rehovot, Israel. drilanmedrev@gmail.com 
ORCID ID - 0000-0003-3605-5815

RUNNING TITLE:

\section{CHILDHOOD ALLERGY LINKED WITH ADHD/ASD}

\section{WORD COUNT:}

* Abstract -218 words

* Main Text - 2912 words

NUMBER OF TABLES AND FIGURES:

* Tables -4

* Figures - 3CONFLICT OF INTEREST: The Authors whose names are listed below, declare that there is no conflict of interest in the subject matter or materials discussed in this manuscript.

FINANCIAL SUPPORT: The work was partially funded by a grant from the Israel Ambulatory Pediatric Association.

\section{ABSTRACT:}

Background: Previous studies reported controversial results regarding the association between allergic disorders and ADHD/ASD. The aim of this article is to investigate whether allergic disorders are associated with $\mathrm{ADHD} / \mathrm{ASD}$ in a large cohort of pediatric patients.

Methods: A retrospective study using the pediatric (0-18 year) database (ICD-9-CM codes) of Clalit Health Services during the years (2000-2018). Diagnosis of all disorders was made by specialist physicians.

Results: 117,022 consecutive non-selective allergic children diagnosed with one or more allergic disorder (asthma, rhinitis. conjunctivitis, skin, food, or drug allergy) and 116,968 non-allergic children were enrolled to our study. The mean follow-up period was $11 \pm 6$ years. The presence of allergic disorders in early childhood (mean age of allergic diagnosis $4.5 \pm 4.3$ years) in boys as well as in girls, significantly increased the risk to develop ADHD (O.R 2.45, CI 2.39-2.51; P<0.0001), ASD (O.R 1.17, CI 1.08-1.27; P $<0.0001$ ) or both ADHD + ASD (O.R 1.5, CI 1.35-1.79; $\mathrm{P}<0.0001)$. Children with more than one allergic comorbidity revealed a much higher risk. In a multivariable analysis (adjusted for age at study entry, number of yearly visits and gender) the risk of allergic children to develop ADHD and ADHD + ASD, but not ASD alone, remained significantly higher.

Conclusion: Allergic disorder in early childhood significantly increased the risk to develop ADHD, and to a less extend ASD, in later life.

\section{KEYWORDS:}

pediatric allergic disorders, risk factor, ADHD, ASD, gender.

\section{INTRODUCTION}

Allergic disorders including allergic dermatological diseases especially atopic dermatitis (AD), rhinoconjunctivitis, asthma, food, and drug allergies are common chronic morbidities in pediatric patients. The prevalence of allergic diseases is constantly increasing in the last decades especially in high developed countries. ${ }^{1}$ Though the exact pathogenesis of allergic disorders is not yet defined, it appears that genetic and environmental factors play a role in their development. ${ }^{2}$ In addition to physical discomfort, chronic allergic disorders in early childhood may cause mental and behavioral problems. ${ }^{3}$ Attention deficit hyperactivity disorder (ADHD) is a common neurobehavioral disorder characterized by inattention and hyperactivity that appear before to age of $12 .{ }^{4}$ The prevalence of ADHD in American population (<18 years) is about 9-12\%. ${ }^{5}$ A study from Israel reported ADHD prevalence of $15.2 \% .{ }^{6}$ Autism spectrum disorder (ASD) is a complex neurodevelopmental disorder characterized by deficits in social interaction, language communication with 
repetitive problems. ${ }^{4}$ Its prevalence of in children is about $2.47 \%$, in the $\mathrm{USA},{ }^{7}$ whereas an Israeli study reported a prevalence of $0.65 \%{ }^{8}$ ADHD as well as ASD are more common in boys compering to girls..$^{5-7}$ In some pediatric patients the symptoms of ADHD and ASD overlap, thus they have features of both disorders. ${ }^{9}$

As was shown for allergic disorders, ${ }^{1}$ the prevalence of ADHD and ASD is steadily increased over the past decades. $^{5-8}$ Furthermore, as was reported for allergic disorders, ${ }^{2}$ genetic and environmental factors were shown to play a role in the pathogenesis of ADHD and ASD. ${ }^{10-13}$ Indeed, several studies did investigate the association between allergic disorders and ADHD and/or ASD with controversial results. ${ }^{14-26}$ Differences in size of the studies, follow-up period and diagnostic criteria which were used in the various studies as well as the specific types of allergic disorders that were investigated are most probably the causes for the conflicting reports.

We, therefore, conducted a very large cohort study (about 240,000 pediatric patients) with a long follow up period (from birth to 18 years of age) of consecutive non-selective allergic (rhinitis, conjunctivitis, asthma, food, drug, and skin allergy) and non-allergic patients in order to define the relationship and the association between allergic disorders (all allergic disorders and each one separately) and the development of ADHD, ASD, or both neurological disorders.

\section{PATIENTS AND METHODS}

\section{Data Sources}

In the present retrospective study, we used the pediatric (0-18 years of age) database of Clalit Health Services which is the largest health care provider in Israel during the years 2000-2018. The database contains comprehensive information of all insured subjects including demographic characterizations, dates and details of all hospitalizations and all clinical visits with diagnosis (for each clinical visit/hospitalization) which were coded according to the International Classification of Diseases, $9^{\text {th }}$ revision, Clinical Modification (ICD-9CD). The follow up period was defined from the date of the first to the date of the first to the date of last clinic visit reported for each patient.

We intend to enroll to the present study a large number of consecutive nonselective pediatric subjects 120,000 subjects with a diagnosis of allergic disease designated as "allergic patients" and 120,000 control subjects without any diagnosis of allergic disease - "non-allergic patients" from that database.

Allergic disease was defined in the present of one (or more) of the following: asthma (ICD-9-CM code 493) diagnosed by pulmonologist, allergist or pediatrician; allergic rhinitis (ICD-9-CM code 477) diagnosed by allergist, pediatrician or otolaryngologist; allergic conjunctivitis (ICD-9-CM code 372) diagnosed by ophthalmologist, allergist or of pediatrician; skin allergy including atopic dermatitis (ICD-9-CD code 691,692,708) diagnosed by dermatologist, allergist, or pediatrician; food allergy (ICD-9 CM code 693) diagnosed by allergist; drug allergy (ICD-9 CM code 995) diagnosed by allergist or pediatrician. ADHD (ICD-9-CM code 314) and ASD (ICD-9-CM code 299) where diagnosed by pediatric psychiatrics or pediatric neurologists according to the current version of the diagnostic and statistical manual of mental disorders (the DSM-5). ${ }^{27}$

\section{ETHICAL APPROVAL:}

The study was approved by the Clalit Health Services Ethics Committee in Israel, and was conducted in accordance with all rules of the Declaration of Helsinki.

Statistical analysis

Data is presented as mean \pm standard deviation (SD). For between group comparisons the independent t-test was used for continuous variables and the Pearson Chai-squared test for categorical variables. We performed, first, univariable analysis to calculate the unadjusted odds ratios (OR) and the $95 \%$ confidence intervals (CI) of allergic patients to develop ADHD, ASD or both. (Tables 1, 2, 3; Figure 1). To control for possible confabulations, a multivariate logistic regression analysis adjusting for age at study entry, number of yearly visits per patient and gender was performed (Model 1), (Table 4). A two-tailed p-value equal or less 
than 0.05 was considered statistically significant. All data processing and statistical analysis were performed with Statistical Package for Social Science (SPSS 27).

\section{RESULTS}

234,170 pediatrics patients $(119,874$ males, 114,323 females) were enrolled to our study. During the time of the study (2000-2018), a total of 23,690,318 clinical visits (including hospitalizations) were recorded. The mean $( \pm S D)$ number of yearly visits per patient was $10.3 \pm 10.7$ and the mean $( \pm S D)$ follow-up period, for all enrolled patients was $11 \pm 06$ (range 2-18) years.

117,022 patients were diagnosed with at least one allergic disorder during the time of the study ("allergic patients") whereas the other 116,968 control patients were not diagnosed with any allergic disorder ("nonallergic patients"). As can be seen in Table 1, allergic skin diseases, conjunctivitis, and asthma, were the most prevalent allergic disorders $(49.6 \%, 41.5 \%$ and $32.8 \%$, respectively). About $40 \%$ of our allergic patients had two or more types of allergic disorders (Table 1). During the years of the study, 33,008 of our patients (14\%) where diagnosed with ADHD, 2341 (0.99\%) with ASD and 816 (0.34\%) with both, ADHD and ASD (Table 1).

As can been seen in Table 1, significantly more pediatric patients with a diagnosis of at least one allergic disorder, compared to the non-allergic group of patients, were also diagnosed with ADHD (19.3\% Vs. 8.9\%; $\mathrm{P}<0.0001)$, with ASD (1.1\% Vs. $0.9 \%$; $\mathrm{P}<0.0001)$ or with both, ADHD and ASD $(0.4 \%$ Vs. $0.3 \% ; \mathrm{P}<0.0001)$. The vast majority of those patients (95\% of ADHD and $97 \%$ of ASD patients) were diagnosed with one or more allergic disorders prior to their neurological diagnosis. Furthermore, the mean age of the patients at the time of their first allergic disorder diagnosis ( $4.5 \pm 4.3$ years) was lower than the ages of ADHD (8.5 \pm 3.4 years), ASD (5.1 \pm 3.5 years) and both ADHD and ASD (5.0 \pm 2.9 years) diagnosis. Therefore, we investigated whether allergic disorder in early childhood is associated with the development of ADHD, ASD or both disorders in later life. The presence of any (one or more) allergic disorder significantly increased the risk of those pediatric patients to develop ADHD (OR 2.25; 95\% CI 2.39-2.51), ASD (OR 1.17; 95\% CI 1.08-1.27) or both, ADHD and ASD (OR 1.56; 95\% CI 1.35-1.79) (Table 1). Interestingly, the mean age at the time of ADHD or ASD diagnosis was similar in the allergic and non-allergic group of patients (Table 1).

Table 2 clearly demonstrates that any allergic disorder (evaluated separately) significantly increased the risk of the develop ADHD as compared to the risk observed in non-allergic patients. The early diagnosis of rhinitis and conjunctivitis was associated with the highest risk to develop ADHD (OR 3.958; 95\% CI 3.801-4.122 for rhinitis and OR 3.36; 95\% CI 3.53-3.74 for allergic conjunctivitis). Early diagnosis of skin, drug and especially food allergy were associated with a significant risk to develop ASD. All types of allergic disorders were significantly associated with an increased risk to develop both, ADHD and ASD, except for drug allergy that almost reached statistical significance (Table 2).

We further investigated the association between early diagnosis of allergic disorders and the development of ADHD, ASD or both neurological disorders. To this end, we studied the effect of the number of allergic disorders (for each patient) on the risk to develop ADHD or ASD. As can be seen in Figure 1A, the risk to develop ADHD, significantly increased in patients with several allergic comorbidities. Thus, a patient with five or six allergic disorders demonstrated higher risk to develop ADHD as compared to patients without any allergic disorder or with only one allergic disease. Similar significant results were observed for allergic patients that develop both disorders (ADHD +ASD) (Figure 1C). The association between the number of allergic disorders and the development of ASD was not significant except for patients with six allergic comorbidities that demonstrated significant high OR as compared to the other patients (Figure 1B)

More boys (53.4\%) than girls were recruited to our study. Furthermore, both ADHD and ASD are more common in boys. ${ }^{5-7}$ Therefore, we further evaluated the impact of gender on the association between allergic disorders and the development of ADHD and ASD. Indeed, as can be seen in Table 3, more boys than girls in our study were diagnosed with ADHD and ASD regardless to the presence of any allergic disorder. Nevertheless, the OR to develop ADHD or ADHD with ASD were similar in both genders. The OR to develop ASD alone was significant for boys (1.13; CI 1.03-1.24) but it did not reach statistical significance 
in allergic girls (Table 3).

To ensure that allergy in early childhood is an independent risk factor for the development of ADHD, ASD or both disorders, we further preformed a logistic regression multivariate analysis adjusted for gender, age at study entry and the number of yearly visits per patient (Model 1). As can be seen in Table 4, the presence of allergy was shown to be an independent risk factor to develop ADHD (OR 2.08; 95\% CI 2.03-2.15) or the combination of both, ADHD and ASD (OR 1.19; 95\% CI 1.02-1.38). Although early childhood allergy was shown to be a significant risk factor for ASD development univariable analysis (Tables 1, 4), in the multivariable analysis (Model 1), it did not reach statistical significance (Table 4).

\section{DISCUSSION}

The present study demonstrates significant association between various allergic disorders (rhinitis, conjunctivitis, asthma, drug, and food allergy) in early childhood and the development of ADHD. The association with ASD was less significant. The presence of several allergic comorbidities (in one patient) further increases the risk to develop those neurobehavioral disorders.

In a very large $(117,022)$ cohort of pediatric allergic (with one or more of the following: asthma, rhinitis, conjunctivitis, skin, food, and drug allergy) and non-allergic $(116,968)$ patients from rural and urban communities, we were able to demonstrate significant association between allergic disorders (one or more) in early childhood and the development of ADHD or ASD (Table 1). The OR for ADHD (2.45) and ADHD+ASD (1.56) were higher than for ASD (1.17), but still the later was statistically significant in a univariate analysis. A dose dependent relationship was observed, thus as was previously reported, ${ }^{17,28}$ the risk to develop ADHD or ASD increased in children with several (more than one) allergic comorbidities (Figure 1). The latter, further support the link between allergic disorders and ADHD/ASD. The large number of enrolled patients, the long follow up period (from birth to 18 years of age), the assessment of various allergic comorbidities which were diagnosed by specialist physicians contribute to the strength and validation of our study.

Most previous studies investigated the association of a single allergic disorder, ${ }^{15-17,29-33}$ or up to three allergic comorbidities, ${ }^{14,23,24,26,28,34,35}$ and ADHD/ASD. To the best of our knowledge, the present study is the first study which enrolled children with up to six allergic comorbidities (Table 1) including drug allergy that was not previously studied. Moreover, whereas most previous studies reported the association between allergic disorders and ADHD or ASD, ${ }^{15,16,29,31}$ we investigated the link to ADHD and ASD (or both ADHD+ASD) using our large pediatric cohort, with the same methodological and statistical analysis that add to the validity of our findings.

The presence of each allergic disorder (studied separately) significantly increased the risk to develop ADHD or ADHD $+\mathrm{ASD}$. The fact the association between drug allergy and ADHD +ASD (OR 1.7; 95\% CI 0.95-3.03) did not reached statistical significance is most probably due to the relatively low number of patients with drug allergy that were enrolled to our study. The highest OR was observed for children with rhinitis and conjunctivitis (OR of 1.93; 95\% CI 1.51-2.47 and 1.86; 95\% CI 1.58-2.2; respectively) (Table 2). Although the presence of one or more allergic disorder was significantly associated with the development of ASD (Table 1), when studied separately only skin, food and drug allergy were found to be significant with OR's that were lower compared to the risk (OR) to develop ADHD (Table 2).

The prevalence of ADHD and ASD, diagnosed by pediatric psychiatrics or neurologists, in our study (Table 1,3 ), is similar to previous reports. ${ }^{5-8}$ Moreover, as was previously demonstrated, ${ }^{5-7}$ more boys than girls were diagnosed with ADHD or ASD (Table 3). Allergic boys and girls revealed similar significant risk to developed ADHD or ADHD + ASD, whereas only boys demonstrated significant risk to develop ASD alone (Table 3). Indeed, after adjusting for age at study entry, number of yearly visits per patient and gender (Model 1) (Table 4), the risk of allergic children to develop ADHD or ASD + ADHD as compared to nonallergic children was significantly higher (OR of 2,08 and 1.19; respectively) whereas their risk to develop ASD alone was not significant in the multivariable analysis (Table 4).

Several previous studies did not find significant association between allergic disorders and the development 
of $\mathrm{ADHD}^{15,17}$ or ASD, ${ }^{25,29}$. However, most studies that were done in different parts of the world provide evidence for high risk of pediatric allergic patients to develop $\mathrm{ADHD}^{17,24,28,30-32}$ or $\mathrm{ASD}^{14,17,23,28,32}$ regardless to the ethnicity of the enrolled patients. A meta-analysis by Schans et al, ${ }^{26}$ reported that asthma, rhinitis, and eczema were independent risk factors for ADHD development which is similar to our observation. Interestingly, in contrast to our observations (Tables 1, 2, 3, 4), two studies ${ }^{17,28}$ reported that the risk of allergic children to develop ASD was higher than their risk to develop ADHD. Differences in methodology (e.g. cohort type and size, follow up period and mode of diagnosis: self-report Vs. physician) and in the statistical evaluation are most probably the reason for the conflicting results.

There are several possible mechanisms, not mutually exclusive, for the association between allergic disorders (Table 1, 2, 3, and 4) and ADHD or ASD. First, allergic disorders and ADHD and ASD may have common genetic and environmental factors that increase the co-occurrence of those disorders. The high rates of ASD in children of mothers with allergic disorders ${ }^{36}$ and the increased risk for allergic disorders in siblings of ASD patients, ${ }^{37}$ may support the above mechanism. Indeed, genetic links between allergic diseases and $\mathrm{ASD}^{36}$ or $\mathrm{ADHD}^{10,38}$ were reported. In addition, dysregulation of tryptopan and serotonin metabolism was reported to play a role in the pathogenesis of allergic disorders as well and in $\mathrm{ADHD}^{39}$ and ASD. ${ }^{40}$ The fact that we (Table 1), as well as others ${ }^{17}$ had demonstrated that allergy preceded the development of ADHD or ASD may point to other mechanism(s). Mast cell activation, high levels of inflammatory cytokines (especially Th2 cytokines) and chemokines (CCXL8, CCL2) observed in patients with allergic disorders can cause neuroimmune microglial and mammalian Target of Rapamycin (mTOR) activation, brain inflammation and neurobehavioral disorders. ${ }^{11,13,41,42}$ In addition, stress, fear (especially in food and skin allergy) and sleep disturbances (observed in allergic children) may also play a role in the pathogenesis of ADHD or ASD. Schmitt el al suggested that the usage of antihistamine agents that pass the brain blood barrier (in early childhood) may contribute to the development of the neurobehavioral disorders. ${ }^{16}$

The main limitation of our present study is its retrospective nature rather than a prospective controlled study that may give more valid results. Our study does not provide genetic or environmental data which may be potential confounders. In addition, the prevalence of allergic disorders as well as ADHD or ASD in our study might be underestimated since only those who sought medical services were recruited. Nevertheless, our study presents a very large number of pediatrics patients $(237,170$; Table 1$)$ from urban and rural areas with a long follow up period ( $11 \pm 0.6$ years). Moreover, the diagnosis (allergic, neurological) in our study, was made by specialist physicians, rather than by self or parent reports, leading to a high diagnostic validity. Interestingly, Sohn et al reported recently that individuals with developmental disorders had higher odds of self-reported allergic disease but not of allergic sensitization to foods or environmental allergens. ${ }^{43}$ The fact that the diagnosis of all diseases, allergic as well as ADHAD/ASD, in our study were made by specialist physicians overcome such a confabulation. Finally, we investigated the association between the presence of six allergic disorders (together and each one separately), and the development of ADHD or ASD (Tables $1,2,3,4)$. Taken together, our study provides solid evidence supporting the association between allergic disorders and $\mathrm{ADHD} / \mathrm{ASD}$.

The results of our study have clinical applications for physicians who take care of pediatrics patients. Treatment and reduction of sleep disorders, stress, and all kind of physical discomforts in allergic children, may (tough it is not proved) reduce the development of neurobehavioral disorders such as ADHD and ASD. In addition, knowledge of the link and the association between allergic disorders and the development of ADHD or ASD will lead to early diagnosis and better treatment of allergic children with neurobehavioral symptoms.

In conclusion, our study provides strong evidence for the association between allergic disorders in early childhood and the development of ADHD. The risk of those children to develop ASD was less significant.

\section{ACKNOWLEDGMENTS:}

The authors would like to express their appreciation to Ms. Ronit Harris, Statistics Consultant, for her assistance in statistical analysis of the data for this paper.

KEY MESSAGE: 
Early and effective treatment of allergic disorders, including sleep disorders, stress, and all types of physical discomforts found in allergic children, may reduce the development of neurobehavioral disorders such as ADHD and ASD.

\section{REFERENCES}

1. Gupta R, Sheikh A, Strachan DP, Anderson HR. Burden of allergic disease in the UK: secondary analyses of national databases. Clin Exp Allergy . 2004;34(4):520-526. doi:10.1111/j.1365-2222.2004.1935.x

2. Vickery BP, Chin S, Burks AW. Pathophysiology of food allergy.Pediatr Clin North Am . 2011;58(2):36376, ix. doi:10.1016/j.pcl.2011.02.012

3. Tzeng N-S, Chang H-A, Chung C-H, et al. Increased Risk of Psychiatric Disorders in Allergic Diseases: A Nationwide, Population-Based, Cohort Study. Front Psychiatry . 2018;9:133. doi:10.3389/fpsyt.2018.00133

4. Miyasaka M, Kajimura S, Nomura M. Biases in understanding attention deficit hyperactivity disorder and autism spectrum disorder in japan.Front Psychol . 2018;9:244. doi:10.3389/fpsyg.2018.00244

5. Visser SN, Danielson ML, Bitsko RH, et al. Trends in the parent-report of health care provider-diagnosed and medicated attention-deficit/hyperactivity disorder: United States, 2003-2011.J Am Acad Child Adolesc Psychiatry . 2014;53(1):34-46.e2. doi:10.1016/j.jaac.2013.09.001

6. Brook U, Boaz M. Attention deficit and learning disabilities (ADHD/LD) among high school pupils in Holon (Israel). Patient Educ Couns . 2005;58(2):164-167. doi:10.1016/j.pec.2004.07.012

7. Xu G, Strathearn L, Liu B, Bao W. Prevalence of Autism Spectrum Disorder Among US Children and Adolescents, 2014-2016. JAMA . 2018;319(1):81-82. doi:10.1001/jama.2017.17812

8. Davidovitch M, Hemo B, Manning-Courtney P, Fombonne E. Prevalence and incidence of autism spectrum disorder in an Israeli population. J Autism Dev Disord . 2013;43(4):785-793. doi:10.1007/s10803-012-1611-z

9. Avni E, Ben-Itzchak E, Zachor DA. The presence of comorbid ADHD and anxiety symptoms in autism spectrum disorder: clinical presentation and predictors. Front Psychiatry . 2018;9:717. doi:10.3389/fpsyt.2018.00717

10. Tylee DS, Sun J, Hess JL, et al. Genetic correlations among psychiatric and immune-related phenotypes based on genome-wide association data. Am J Med Genet B, Neuropsychiatr Genet . 2018;177(7):641-657. doi:10.1002/ajmg.b.32652

11. Marshall P. Attention deficit disorder and allergy: a neurochemical model of the relation between the illnesses. Psychol Bull . 1989;106(3):434-446. doi:10.1037/0033-2909.106.3.434

12. Ashwood P, Krakowiak P, Hertz-Picciotto I, Hansen R, Pessah I, Van deaaa 1Water J. Elevated plasma cytokines in autism spectrum disorders provide evidence of immune dysfunction and are associated with impaired behavioral outcome. Brain Behav Immun . 2011;25(1):40-45. doi:10.1016/j.bbi.2010.08.003

13. Theoharides TC, Tsilioni I, Patel AB, Doyle R. Atopic diseases and inflammation of the brain in the pathogenesis of autism spectrum disorders. Transl Psychiatry . 2016;6(6):e844. doi:10.1038/tp.2016.77

14. Xu G, Snetselaar LG, Jing J, Liu B, Strathearn L, Bao W. Association of food allergy and other allergic conditions with autism spectrum disorder in children. JAMA Netw Open . 2018;1(2):e180279. doi:10.1001/jamanetworkopen.2018.0279

15. Johansson EK, Ballardini N, Kull I, Bergström A, Wahlgren C-F. Association between preschool eczema and medication for attention-deficit/hyperactivity disorder in school age. Pediatr Allergy Immunol . 2017;28(1):44-50. doi:10.1111/pai.12657

16. Schmitt J, Buske-Kirschbaum A, Tesch F, et al. Increased attention-deficit/hyperactivity symptoms in atopic dermatitis are associated with history of antihistamine use. Allergy . 2018;73(3):615-626. doi: $10.1111 /$ all.13326 
17. Chen M-H, Su T-P, Chen Y-S, et al. Is atopy in early childhood a risk factor for ADHD and ASD? a longitudinal study. J Psychosom Res . 2014;77(4):316-321. doi:10.1016/j.jpsychores.2014.06.006

18. Bakkaloglu B, Anlar B, Anlar FY, et al. Atopic features in early childhood autism. Eur J Paediatr Neurol . 2008;12(6):476-479. doi:10.1016/j.ejpn.2007.12.008

19. Biederman J, Milberger S, Faraone SV, Guite J, Warburton R. Associations between childhood asthma and ADHD: issues of psychiatric comorbidity and familiality. $J$ Am Acad Child Adolesc Psychiatry . 1994;33(6):842-848. doi:10.1097/00004583-199407000-00010

20. Mogensen N, Larsson H, Lundholm C, Almqvist C. Association between childhood asthma and ADHD symptoms in adolescence-a prospective population-based twin study. Allergy . 2011;66(9):1224-1230. doi:10.1111/j.1398-9995.2011.02648.x

21. Schmitt J, Romanos M, Schmitt NM, Meurer M, Kirch W. Atopic eczema and attentiondeficit/hyperactivity disorder in a population-based sample of children and adolescents. JAMA . 2009;301(7):724-726. doi:10.1001/jama.2009.136

22. Chen M-H, Su T-P, Chen Y-S, et al. Comorbidity of allergic and autoimmune diseases among patients with ADHD. J Atten Disord . 2017;21(3):219-227. doi:10.1177/1087054712474686

23. Hori D, Tsujiguchi H, Kambayashi Y, et al. The Association of Autism Spectrum Disorders and Symptoms of Asthma, Allergic Rhinoconjunctivitis and Eczema among Japanese Children Aged 3 - 6 Years. Health (Irvine, Calif) . 2017;09(08):1235-1250. doi:10.4236/health.2017.98089

24. Tsai J-D, Chang S-N, Mou C-H, Sung F-C, Lue K-H. Association between atopic diseases and attention-deficit/hyperactivity disorder in childhood: a population-based case-control study. Ann Epidemiol . 2013;23(4):185-188. doi:10.1016/j.annepidem.2012.12.015

25. Akpınar F, Kutluk G, Özomay G, Yorbik Ö, Çetinkaya F. Frequencies of Allergic Diseases Among Children with Autism Spectrum Disorders.Asthma Allergy Immunol . Published online January 5, 2019. doi:10.21911/aai.170

26. Schans J van der, Çiçek R, de Vries TW, Hak E, Hoekstra PJ. Association of atopic diseases and attention-deficit/hyperactivity disorder: A systematic review and meta-analyses. Neurosci Biobehav Rev . 2017;74(Pt A):139-148. doi:10.1016/j.neubiorev.2017.01.011

27. Doernberg E, Hollander E. Neurodevelopmental Disorders (ASD and ADHD): DSM-5, ICD-10, and ICD-11. CNS Spectr . 2016;21(4):295-299. doi:10.1017/S1092852916000262

28. Lee C-Y, Chen M-H, Jeng M-J, et al. Longitudinal association between early atopic dermatitis and subsequent attention-deficit or autistic disorder: A population-based case-control study. Medicine . 2016;95(39):e5005. doi:10.1097/MD.0000000000005005

29. Jónsdóttir U, Lang JE. How does autism spectrum disorder affect the risk and severity of childhood asthma? Ann Allergy Asthma Immunol . 2017;118(5):570-576. doi:10.1016/j.anai.2017.02.020

30. Genuneit J, Braig S, Brandt S, et al. Infant atopic eczema and subsequent attention-deficit/hyperactivity disorder-a prospective birth cohort study. Pediatr Allergy Immunol . 2014;25(1):51-56. doi:10.1111/pai.12152

31. Atefi N, Rohaninasab M, Shooshtari M, et al. The Association between Attention-Deficit/Hyperactivity Disorder and Atopic Dermatitis: A Study among Iranian Children. Indian J Dermatol . 2019;64(6):451-455. doi:10.4103/ijd.IJD_458_18

32. Liao T-C, Lien Y-T, Wang S, Huang S-L, Chen C-Y. Comorbidity of Atopic Disorders with Autism Spectrum Disorder and Attention Deficit/Hyperactivity Disorder. J Pediatr . 2016;171:248-255. doi:10.1016/j.jpeds.2015.12.063 
33. Nygaard U, Riis JL, Deleuran M, Vestergaard C. Attention-Deficit/Hyperactivity Disorder in Atopic Dermatitis: An Appraisal of the Current Literature. Pediatr Allergy Immunol Pulmonol . 2016;29(4):181188. doi:10.1089/ped.2016.0705

34. van der Schans J, Cao Q, Bos EH, et al. The temporal order of fluctuations in atopic disease symptoms and attention-deficit/hyperactivity disorder symptoms: a time-series study in ADHD patients. Eur Child Adolesc Psychiatry . 2020;29(2):137-144. doi:10.1007/s00787-019-01336-2

35. Chang HY, Seo J-H, Kim HY, et al. Allergic diseases in preschoolers are associated with psychological and behavioural problems.Allergy Asthma Immunol Res . 2013;5(5):315-321. doi:10.4168/aair.2013.5.5.315

36. Croen LA, Qian Y, Ashwood P, et al. Family history of immune conditions and autism spectrum and developmental disorders: Findings from the study to explore early development. Autism Res . 2019;12(1):123135. doi:10.1002/aur.1979

37. Dai Y-X, Tai Y-H, Chang Y-T, Chen T-J, Chen M-H. Increased Risk of Atopic Diseases in the Siblings of Patients with Autism Spectrum Disorder: A Nationwide Population-Based Cohort Study. J Autism Dev Disord . 2019;49(11):4626-4633. doi:10.1007/s10803-019-04184-w

38. Solberg BS, Zayats T, Posserud M-B, et al. Patterns of Psychiatric Comorbidity and Genetic Correlations Provide New Insights Into Differences Between Attention-Deficit/Hyperactivity Disorder and Autism Spectrum Disorder. Biol Psychiatry . 2019;86(8):587-598. doi:10.1016/j.biopsych.2019.04.021

39. Banerjee E, Nandagopal K. Does serotonin deficit mediate susceptibility to ADHD? Neurochem Int . 2015;82:52-68. doi:10.1016/j.neuint.2015.02.001

40. Abdulamir HA, Abdul-Rasheed OF, Abdulghani EA. Serotonin and serotonin transporter levels in autistic children. Saudi Med J . 2018;39(5):487-494. doi:10.15537/smj.2018.5.21751

41. Pelsser LMJ, Buitelaar JK, Savelkoul HFJ. ADHD as a (non) allergic hypersensitivity disorder: a hypothesis. Pediatr Allergy Immunol . 2009;20(2):107-112. doi:10.1111/j.1399-3038.2008.00749.x

42. Kalkman HO, Feuerbach D. Microglia M2A Polarization as Potential Link between Food Allergy and Autism Spectrum Disorders.Pharmaceuticals (Basel) . 2017;10(4). doi:10.3390/ph10040095

43. Sohn JK, Keet CA, McGowan EC. Association between allergic disease and developmental disorders in the National Health and Nutrition Examination Survey. J Allergy Clin Immunol Pract . 2019;7(7):2481-2483.e1. doi:10.1016/j.jaip.2019.04.013

\section{LEGEND TO FIGURE 1}

The risk to develop ADHD (A), ASD (B) or both ADHD and ASD (C) in non-allergic patients (0 allergic comorbidities) was defined as 1 . Concomitantly with the number of allergic comorbidities per patient (from 1 to 6), the OR to develop ADHD (A) or ADHD and ASD (C) also increased significantly. The number of allergic comorbidities per patient did not reveal significant effect on the risk to develop ASD (B). Allergic comorbidities per Table 1 (Asthma, rhinitis, conjunctivitis, skin, food and drug allergy).

\section{Hosted file}

Figures and Tables.docx available at https://authorea.com/users/453647/articles/551434-earlychildhood-allergy-linked-with-development-of-attention-deficit-hyperactivity-disorderand-autism-spectrum-disorder 